\title{
Epicardium-to-fat transition in injured heart
}

\author{
Cell Research (2014) 24:1367-1369. doi:10.1038/cr.2014.125; published online 26 September 2014
}

\section{Dear Editor,}

The origin of fat cells or adipocytes is a fundamental biological question with important ramifications for human health and disease [1]. Epicardial fat is associated with increased risk of cardiovascular diseases such as coronary atherosclerosis [2]. However, the origin of these epicardial fat cells remains largely unknown. Epicardial progenitors play a pivotal role in the developing heart, by secreting paracrine signals and by differentiating into fibroblasts, smooth muscle cells, and potentially endothelial cells and cardiomyocytes [3-6]. Some of these developmental programs are reactivated during postnatal heart injury $[7,8]$, and reactivated adult epicardial progenitors provide pro-angiogenic protection and undergo limited epithelial-to-mesenchymal transition in cardiac injury $[7,9]$. Since epicardial fat is physically adjacent to epicardial cells, we hypothesized that some of these cells originate from the epicardium during development and in postnatal heart injury.

We first generated Wt1-CreER; Rosa26 $6^{\mathrm{RFP} /+}$ double transgenic mouse line. In the absence of tamoxifen, we found little detectable labeling of epicardial progenitors and their derivatives [7]. After tamoxifen induction, translocation of CreER into nucleus allowed removal of a loxP-flanked transcriptional stop cassette, thus leading to indelible RFP labeling of epicardial cells and their descendants, epicardium-derived cells (EPDCs, Supplementary information, Figure S1A). We injected tamoxifen at embryonic day 10.5 (E10.5) to label epicardial progenitors and collected hearts during postnatal weeks 8 to 12 (P8w to P12w) to analyze their contribution to epicardial fat (Figure 1A). We found that fetal EPDCs contribute significantly to intramyocardial vessels in adult hearts (Supplementary information, Figure S1A). Interestingly, some EPDCs, particularly those in the atrioventricular groove, remained in an epicardial location and colocalized with fat tissue (Figure 1B). Co-immunostaining of RFP and adipocyte marker perilipin (PLIN) in tissue sections from four hearts (12 sections each) verified that among $3560 \mathrm{PLIN}^{+}$fat cells counted, $23.4 \% \pm 5.1 \%$ were RFP $^{+}$cells (Figure 1C).

To further confirm this finding, we employed an ade- novirus that expresses Cre under the epicardium-specific promoter Msln to selectively lineage-trace the epicardium [7]. Ad:Msln-Cre transduction of cultured epicardial cells activated expression of GFP from a Cre-dependent Rosa $26^{\mathrm{mTmG} /+}$ reporter (Supplementary information, Figure S1B). As Msln is specifically expressed in epicardium of the developing heart (Supplementary information, Figure S1C), we performed ultrasound-guided embryonic heart injection of Msln-Cre virus at E12.5. Examination of E15.5 hearts confirmed selective labeling of the epicardium (Supplementary information, Figure S1D). We next collected P8w hearts and found that a subset of Msln-Cre-labeled EPDCs co-expressed PLIN, demonstrating contribution of epicardial progenitors to fat cells during normal heart development (Figure 1D). Altogether, the above data showed that embryonic epicardial progenitors contribute to epicardial fat. We refer to this process as epicardium-to-fat transition (EFT).

From this intriguing observation in heart development, we next asked whether adult epicardial progenitors contribute to epicardial fat cells. We first performed adult epicardial lineage tracing by tamoxifen injection in an 8 -week-old adult Wt1-CreER; Rosa26 ${ }^{\mathrm{RFP} /+}$ mice. A significant portion of $\mathrm{WT}^{+}$epicardial progenitors in the atrioventricular groove were labeled after 4-6 weeks (Supplementary information, Figure S1E). However, we did not detect any $\mathrm{RFP}^{+}$epicardial fat cells (Supplementary information, Figure S1F), suggesting that adult epicardial progenitors do not actively generate epicardial fat during normal heart homeostasis. This was not simply due to limited tamoxifen-induced labeling by Wt1-CreER, as a significant portion of epicardial cells were labeled in the vicinity of epicardial adipose tissue (Supplementary information, Figure S1E and S1F). In addition, labeling of renal cells in the kidneys of the same mice was high (Supplementary information, Figure S1E). Taken together, the above data suggested that while embryonic epicardial cells contribute to epicardial adipose tissue, there is minimal EFT in normal adult heart.

Our previous work shows that postnatal epicardial cells are a dynamic progenitor population that reactivates fetal properties after injury $[7,9,10]$. To directly address the lineage conversion of epicardial progenitors 
A

Wt1-CreER Rosa26 ${ }^{\text {RPP/t }}$

Tam

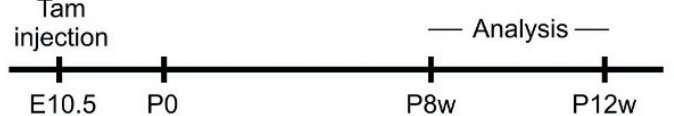

B

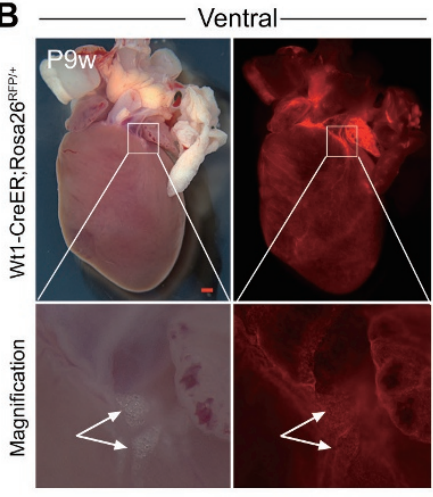

C

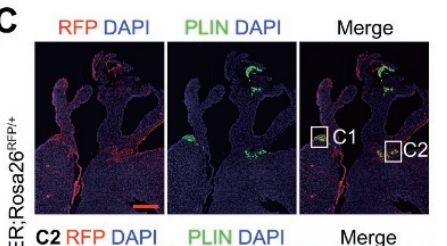

(ै) C2RFP DAPI PLIN DAPI

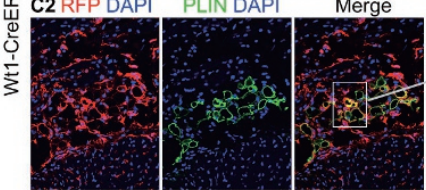

D



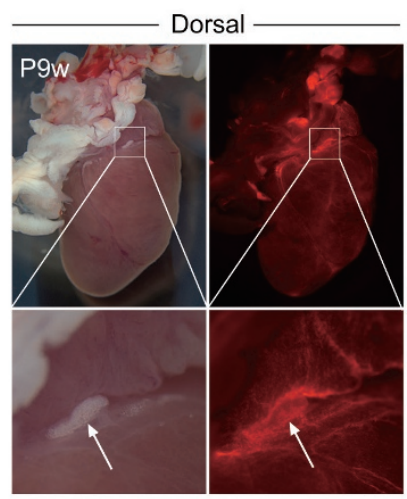
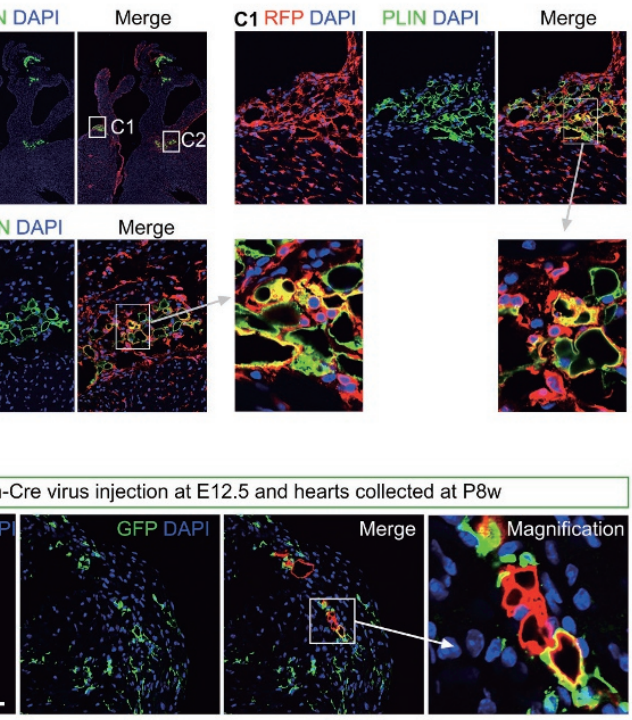

E

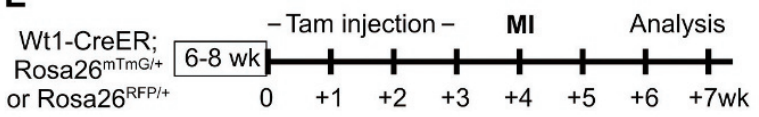

$\mathbf{F}$
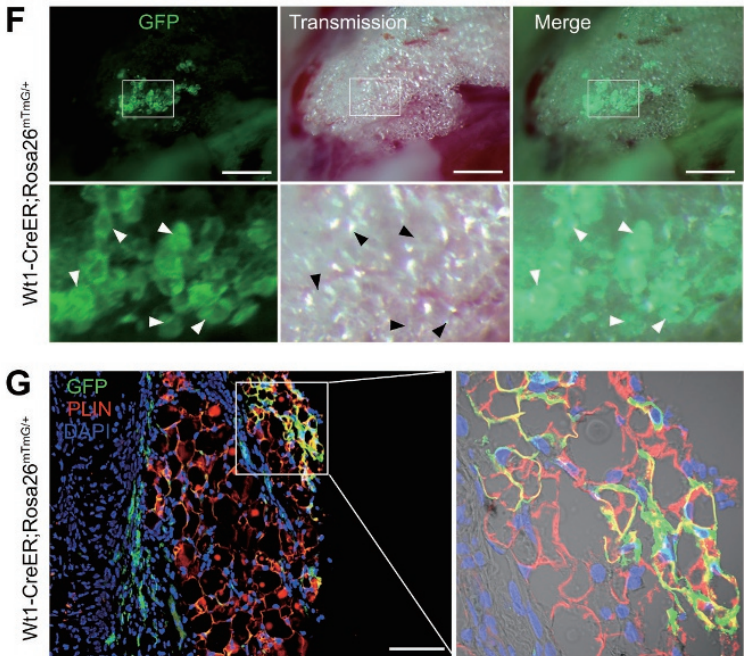

H Msln-Cre virus injection at P8w before Ml and hearts collected at P12w



PLIN DAPI

GFP DAPI

Merge

Magnification
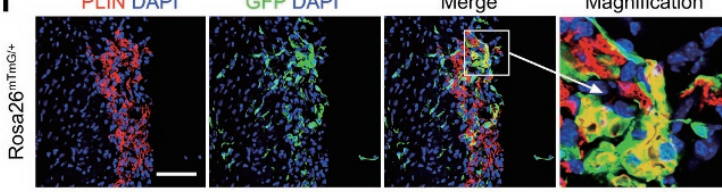

Figure 1 Epicardial cells differentiate into adipocytes during cardiac development and injury. (A) Strategy of inducible labeling of epicardial cells. Tamoxifen was administered at E10.5 to Wt1-CreER; Rosa26 ${ }^{\mathrm{RFP} /+}$ embryos, and adult hearts were collected for analysis. (B) Whole-mount view of fat tissue in atrioventricular groove (arrows). (C) Sections stained for lineage marker RFP, adipocyte marker PLIN, and nuclei marker DAPI. C1 and C2 show that a subset of adipocytes in the right and left atrioventricular grooves were derived from epicardial cells. Images are representative of results from four individual hearts. (D) Co-immunostaining of PLIN and GFP in P8w Rosa26 ${ }^{\mathrm{mTm} / \mathrm{+}}$ heart treated with Msln-Cre adenovirus at E12.5. (E) Strategy for lineage tracing of adult epicardial cells after MI. Adult mice were treated with tamoxifen before MI. (F) Whole-mount picture of adult labeled heart following MI. Arrowheads indicate epicardium-derived adipocytes within fat tissue on heart surface. (G) Immunostaining of post-MI heart sections shows that a subset of PLIN ${ }^{+}$adipocytes co-express the GFP lineage marker. (H) MsIn-Cre adenovirus was injected into the adult heart. After MI, whole-mount images show lineage-traced GFP ${ }^{+}$cells within fat tissue on the heart surface. (I) Immunostaining of PLIN and GFP in MsIn-Cre adenovirus-treated Rosa26 ${ }^{\mathrm{mTmG} /+}$ hearts after MI. Red bar $=0.5 \mathrm{~mm}$; yellow bar $=200 \mu \mathrm{m}$; white bar $=100 \mu \mathrm{m}$.

to fat cells after cardiac injury, we first lineage-tagged epicardium by tamoxifen induction of Wt1-CreER; Rosa $26^{\mathrm{mTmG} /+}$ hearts, and then performed myocardial infarction (MI) by ligation of the left anterior descending coronary artery (Figure 1E). Since WT1 is specifically expressed in epicardium but not fat cells (Supplementary information, Figure S1G), epicardium was specifically prelabeled before MI (Supplementary information, Figure $\mathrm{S} 1 \mathrm{H})$. Three weeks after MI, we collected the injured hearts with attached fat tissue on their surface, and found 
that a subset of epicardial fat cells near the border zone of infarcted myocardium was genetically labeled (Figure $1 \mathrm{~F})$. We confirmed that these labeled cells were adipocytes by co-staining of PLIN and GFP (Figure 1G). Adult epicardial cells contributed to $9.3 \% \pm 3.7 \%$ of fat cells in epicardial adipose tissue in the peri-infarct region ( $n$ $=4$, Figure $1 \mathrm{G}$ ). We verified this result by serial sections from Wt1-CreER; Rosa26 ${ }^{\mathrm{RFP} /+}$ MI hearts (Supplementary information, Figure S1I). To show that cell labeling was not due to CreER activity in the absence of the inducing agent under MI stress conditions, we examined Wt1CreER; Rosa $26^{\mathrm{mTmG} /+}$ MI hearts that were not treated with tamoxifen and found no $\mathrm{GFP}^{+}$epicardial fat cells. MI also induced proliferation of adipocytes after MI, suggesting that adipocyte expansion as well as EFT contribute to expansion of epicardial fat after MI (Supplementary information, Figure S1J).

To verify this finding, we injected Msln-Cre virus into adult Rosa $26^{\mathrm{mTmG} /+}$ heart before MI and confirmed epicardial labeling in pre-MI hearts (Supplementary information, Figure S1K). We then performed MI on these Msln-Cre-injected mice, and collected the hearts 4 weeks afterwards. We found that a subset of cells in epicardial fat tissue were expressing GFP, suggesting that labeled epicardial cells contribute to adipocytes in injured heart (Figure 1H). By co-immunostaining of PLIN and GFP, we confirmed $\mathrm{GFP}^{+} \mathrm{PLIN}^{+}$fat cells in MI heart (Figure 1I), suggesting that these epicardial fat cells were derived from Msln-Cre-prelabeled epicardial progenitors.

EPDCs have been reported to differentiate into multiple cell types, most recently adipocytes [11]. To ask whether single EPDC is multipotent, we performed clonal assays on EPDCs isolated from MI hearts and expanded clonally from single cells. We FACS sorted EPDCs from Wt1CreER; Rosa $26^{\mathrm{mTmG} / \mathrm{t}}$ hearts 1 week after MI and generated single cell clonal outgrowths. FACS analysis of these clones confirmed that they phenotypically resemble mesenchymal stem cells (Supplementary information, Figure S1L-S1P). When cultured in different conditions, the clonal outgrowths differentiated into adipocyte, chondrocyte, and osteoblast lineages (Supplementary information, Figure S1Q), demonstrating their multipotency.

By genetic lineage tracing, our study revealed that epicardial progenitors contribute to fat cells during development. This EFT potential is quiescent in adult heart, but is reactivated after MI. Our work suggested that the EFT might be a cardiac response to severe injury such as MI, and that EFT could be a new signature of cardiovascular diseases. A recent study showed that mesothelium, including epicardium, contributes to visceral fat [11]. Our study of the epicardium confirmed this result and extended it to homeostasis and diseases of the adult heart. EFT makes limited contributions to epicardial adipose tissue during heart homeostasis. However, this potential is unlocked under stress such as MI, which reactivates the developmental EFT program and leads to injury-stimulated EFT in vivo. Understanding of the molecular regulatory mechanisms of EFT will provide insights into the treatment of cardiovascular diseases and regenerative medicine $[1,12,13]$.

\section{Acknowledgments}

This work was supported by the Ministry of Science and Technology of China (2012CB945102 and 2013CB945302), the National Natural Science Foundation of China (91339104, 31271552, 31222038), the Chinese Academy of Sciences (Hundred Talents Program; KSCX2-EW-R-09), Organization Department of the CPC Central Committee Bajian Talents Program, Shanghai Pujiang Program (11PJ1411400) and Basic Research Key Project (14JC1407400). WTP was funded by NIH (2 R01 HL094683).

Qiaozhen Liu ${ }^{1}$, Xiuzhen Huang ${ }^{1}$, Jin-Hee $\mathrm{Oh}^{2}$, Ruei-Zeng Lin ${ }^{3}$, Shengzhong Duan ${ }^{1}$, Ying $\mathrm{Yu}^{1}$, Rui Yang ${ }^{4}, J_{u}$ Qiu ${ }^{4}$, Juan M Melero-Martin³, William T Pu ${ }^{5}$, Bin Zhou $^{1}$

${ }^{l}$ Key Laboratory of Nutrition and Metabolism, Institute for Nutritional Sciences, Shanghai Institutes for Biological Sciences, Graduate School of the Chinese Academy of Sciences, Chinese Academy of Sciences, Shanghai 200031, China; ${ }^{2}$ Department of Pediatrics, The Catholic University of Korea, Seoul, Korea; ${ }^{3}$ Department of Cardiac Surgery, Boston Children's Hospital, and Department of Surgery, Harvard Medical School, Boston, MA 02115, USA; ${ }^{4}$ Institute of Health Sciences, Shanghai Institutes for Biological Sciences, Chinese Academy of Sciences \& Shanghai Jiao Tong University School of Medicine, Shanghai 200025, China, ${ }^{5}$ Harvard Stem Cell Institute, Harvard University, Cambridge, MA 02138, and Department of Cardiology, Boston Children's Hospital, 300 Longwood Avenue, Boston, MA 02115, USA

Correspondence: Bin Zhou

E-mail: zhoubin@sibs.ac.cn

\section{References}

1 Tang W, Zeve D, Suh J, et al. Science 2008; 322:583-586.

2 Salgado-Somoza A, Teijeira-Fernandez E, Fernandez AL, et al. Atherosclerosis 2012; 224:492-499.

3 Cai C, Martin J, Sun Y, et al. Nature 2008; 454:104-108.

4 Zhou B, Ma Q, Rajagopal S, et al. Nature 2008; 454:109-113.

5 Katz TC, Singh MK, Degenhardt K, et al. Dev Cell 2012; 22:639-650.

6 Lavine K, White A, Park C, et al. Genes Dev 2006; 20:1651-1666.

7 Zhou B, Honor L, He H, et al. J Clin Invest 2011; 121:1894-1904.

8 Bollini S, Vieira JM, Howard S, et al. Stem Cells Dev 2014; 23:1719-1730.

9 Smart N, Bollini S, Dube K, et al. Nature 2011; 474:640-644.

10 Zangi L, Lui KO, von Gise A, et al. Nat Biotechnol 2013; 31:898-907.

11 Chau YY, Bandiera R, Serrels A, et al. Nat Cell Biol 2014; 16:367-375.

12 Aguirre A, Sancho-Martinez I, Izpisua Belmonte JC. Cell Stem Cell 2013; 12:275-284.

13 Masters M, Riley PR. Stem Cell Res 2014; pii: S1873-5061(14)00043-9.

(Supplementary information is linked to the online version of the paper on the Cell Research website.) 